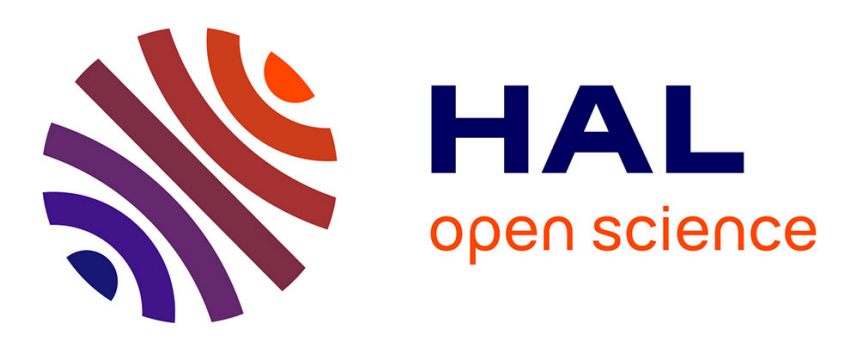

\title{
Combination of Digital Self-Interference Cancellation and AARFSIC for Full-Duplex OFDM Wireless
}

\author{
Zhaowu Zhan, Guillaume Villemaud
}

\section{To cite this version:}

Zhaowu Zhan, Guillaume Villemaud. Combination of Digital Self-Interference Cancellation and AARFSIC for Full-Duplex OFDM Wireless. ICCC 2014, Oct 2014, Shanghai, China. hal-01090702

\section{HAL Id: hal-01090702 https://hal.inria.fr/hal-01090702}

Submitted on 4 Dec 2014

HAL is a multi-disciplinary open access archive for the deposit and dissemination of scientific research documents, whether they are published or not. The documents may come from teaching and research institutions in France or abroad, or from public or private research centers.
L'archive ouverte pluridisciplinaire HAL, est destinée au dépôt et à la diffusion de documents scientifiques de niveau recherche, publiés ou non, émanant des établissements d'enseignement et de recherche français ou étrangers, des laboratoires publics ou privés. 


\title{
Combination of Digital Self-Interference Cancellation and AARFSIC for Full-Duplex OFDM Wireless
}

\author{
Zhaowu Zhan and Guillaume Villemaud \\ Université de Lyon, INRIA \\ INSA-Lyon, CITI-INRIA, F-69621, Villeurbanne, France \\ Email: zhaowu.zhan@insa-lyon.fr
}

\begin{abstract}
Full-Duplex radio provides many benefits beyond improving spectral efficiency. However, canceling the selfinterference completely is a big challenge behind Full-Duplex wireless. Although many intelligent efforts devote to active analog radio frequency self-interference cancellation (AARFSIC), the power of the residual self-interference (SI) after the AARFSIC is still much stronger than that of the receiver thermal noise. In this paper, we first study deeply the AARFSIC and "dig" the core problem that causing the residual SI, and then propose a digital self-interference cancellation in time domain (DSICT) to complement AARFSIC for the Full-Duplex OFDM wireless. The ADS-Matlab co-simulation results demonstrate the actual performance and show that the residual SI after the AARFSIC can be canceled completely by employing the proposed DSICT.
\end{abstract}

\section{INTRODUCTION}

Full-Duplex wireless radio has recently attracted more and more attentions not only from the academic area [1] [2] [3] [4] [5] [6] but also from the industry [7]. Simultaneous transmission and reception in the same frequency band, i.e. Full-Duplex, could greatly improve the spectral efficiency compared to traditional Half-Duplex systems. Beyond improving spectral efficiency, the advantages of Full-Duplex could also be exploited at higher network layers [8]. More generally, Full-Duplex radio could show a great potentiality for any future development of flexible radios.

However, the strong SI behind Full-Duplex challenges the real implementation of Full-Duplex radio. The power of the SI is stronger than that of the signal of interest from the distant radio node by up to $90 \sim 110 \mathrm{~dB}$. Such strong SI could saturate the receiver chain. Therefore, in order to practice the FullDuplex concept, canceling or suppressing the strong SI to noise floor or even under noise floor is the key first step.

The SI could be first suppressed by using the electromagnetic (EM) techniques [5] during the radio wave transmission. Then, active analog radio frequency self-interference cancellation (AARFSIC) [2] [4] [6] could be employed to cancel the SI at the RF stage. Up to date, there is no EM or AARFSIC technique which can bring the SI down to the noise floor. Therefore, it is still necessary to further cancel the SI at the baseband (BB) stage. [9] proposed an active analog $\mathrm{BB}$ self-interference cancellation (AABBSIC) to cancel the SI in the time domain. Another type of baseband self-interference cancellation, digital self-interference cancellation in frequency domain (DSICF), is proposed in [4]. Unfortunately, neither the single technique of these cancellation methods nor the combination of these mechanisms could achieve the required amount of self-interference cancellation (SIC) for the FullDuplex OFDM wireless with multi-path SI channel.

In fact, with respect to the Full-Duplex OFDM wireless with single-path SI channel, the strong SI could be brought down to the noise floor [10] [11] by using AARFSIC [4]. However, we found that the AARFSIC [4] could only achieve very limited amount of SIC for the Full-Duplex OFDM wireless with multipath SI channel, even under the condition that the transceiver RF front-ends are ideal and SI channel state information (CSI) can be obtained perfectly. In the indoor reflecting environment, the AARFSIC can achieve no more than 40dB SIC, order of magnitude was also confirmed by [4].

Contribution: In this paper, we first deeply analyze and study the signal model of the AARFSIC [4] [10] and then dig heavily the core problem causing the residual SI. We discovered that the AARFSIC can not only eliminate the dominant path but also can cancel part of the reflecting paths for Full-Duplex OFDM radio. We also found that the residual SI in time domain is closely related to transmitted time domain symbols, which means the residual SI could be further canceled in the baseband discrete time domain. Based on these facts, we propose to cancel the strong SI by combining the mechanism of AARFSIC [4] [10] and DSICT for Full-Duplex OFDM wireless with multi-path SI channel.

Assumption: In order to highlight the core weakness of the AARFSIC, the transceiver RF front-ends are assumed ideal and the CSI of the SI channel can be obtained perfectly in the theoretical analysis.

\section{Active Analog RF Self-Interference CANCELLATION}

OFDM is a block modulation scheme where a block of $N$ symbols is modulated in parallel on $N$ subcarriers, which can be implemented by using an inverse discrete Fourier transformation (IDFT). This enlightens us that the craft of cancellation signals can be carried out in each subcarrier channel and the SIC can be implemented in the analog RF domain, which we call AARFSIC (illustrated as in Fig. 1). In order to simply the signal model, we just study the local Full-Duplex OFDM radio node, which means the Full-Duplex radio receiver just receives its own transmission.

Let $X_{m, s i}[k], k \in[1, N]$ denote the $m^{t h}$ symbol block which will be modulated by a $N$-size IDFT. The output of 


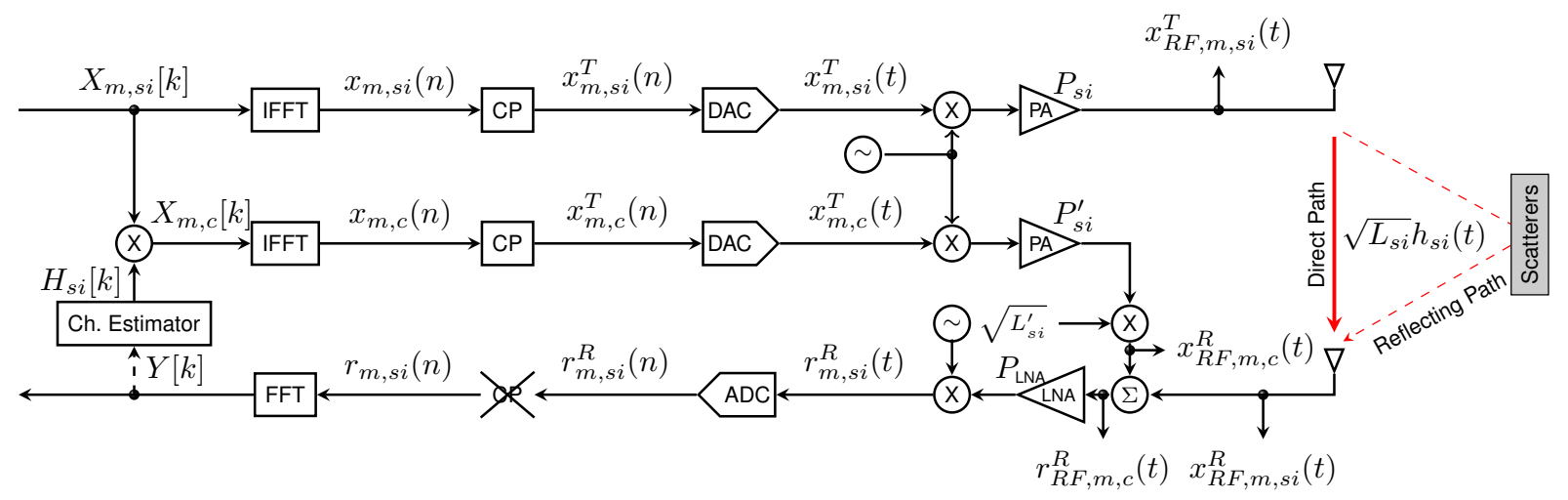

Fig. 1. The block diagram of active analog RF self-interference cancellation.

the IDFT, $x_{m, s i}(n), n \in[1, N]$, is corresponding to the $m^{t h}$ OFDM symbol. After inserting a $N_{\text {guard }}$-length cyclic prefix (CP), the new discrete time domain $\left(N+N_{\text {guard }}\right)$-length OFDM symbol, $x_{m, s i}^{T}(n), n \in\left[1, N+N_{\text {guard }}\right]$, will be converted to the analog signal, $x_{m, s i}^{T}(t)$, by using a digital-to-analog converter (DAC). Then, the baseband analog signal, $x_{m, s i}^{T}(t)$, is upconverted by using local oscillator with carrier frequency $f_{c}$ and amplified by a power amplifier (PA) with power $P_{s i}$, which yields the suitable transmit RF signal $x_{R F, m, s i}^{T}(t)=$ $\sqrt{P_{s i}} x_{m, s i}^{T}(t) e^{j 2 \pi f_{c} t}$. After emitted by the transmit antenna and going through the SI channel $\sqrt{L_{s i}} h_{s i}(t)$ where $L_{s i}$ denotes the path loss of the SI channel, the transmit RF signal $x_{R F, m, s i}^{T}(t)$ is received as RF SI signal, $x_{R F, m, s i}^{R}(t)=$ $\sqrt{L_{s i}} x_{R F, m, s i}^{T}(t) * h_{s i}(t)$, by its own receiver.

In order to implement the AARFSIC to cancel the RF SI, the estimated coefficients, $\widetilde{H}_{s i}[k], k \in[1, N]$, of the SI channel $h_{s i}(t)$ need to be obtained beforehand. With $\widetilde{H}_{s i}[k], k \in[1, N]$ available, we craft a RF cancellation signal, $x_{R F, m, c}^{R}(t)$, in another radio link. In this SI craft link, the $m^{t h}$ new symbol block, $X_{m, c}[k]=X_{m, s i}[k] \widetilde{H}_{s i}[k], k \in[1, N]$ instead of $X_{m, s i}[k], k \in[1, N]$ is modulated by IDFT, which yields a discrete time domain OFDM symbol $x_{m, c}(n), n \in[1, N]$. After adding a $N_{\text {guard }}$-length CP, the $m^{\text {th }}$ cancellation OFDM symbol, $x_{m, c}^{T}(n), n \in\left[1, N+N_{\text {guard }}\right]$, is obtained. DAC is used here to convert the discrete time domain OFDM symbol, $x_{m, c}^{T}(n)$, to continuous time domain OFDM signal, $x_{m, c}^{T}(t)$. Then, another RF front-end is used to up-convert and amplify this baseband signal to match the received RF SI. After the AARFSIC, the residual RF SI, $r_{R F, m, s i}^{R}(t)$, should be the difference between the received RF SI and the cancellation signal. The whole signal processing model of the AARFSIC could be expressed by

$$
\begin{aligned}
r_{R F, m, s i}^{R}(t)= & x_{R F, m, s i}^{R}(t)-x_{R F, m, c}^{R}(t) \\
= & \sqrt{P_{s i}} \sqrt{L_{s i}} \operatorname{DAC}\left\{\operatorname{CP}\left\{\operatorname{IDFT}\left\{X_{m, s i}[k]\right\}\right\}\right\} e^{\jmath 2 \pi f_{c} t} \\
& * h_{s i}(t)-\sqrt{P_{s i}^{\prime}} \sqrt{L_{s i}^{\prime}} \operatorname{DAC}\left\{\operatorname { C P } \left\{\operatorname { I D F T } \left\{X_{m, s i}[k]\right.\right.\right. \\
& \left.\left.\left.\widetilde{H}_{s i}[k]\right\}\right\}\right\} e^{\jmath 2 \pi f_{c} t}
\end{aligned}
$$

where $\operatorname{IDFT}\{\chi[k]\} \triangleq \frac{1}{N} \sum_{k=-\frac{N}{2}}^{\frac{N}{2}-1} \chi[k] e^{\jmath 2 \pi \Delta f k n}$, in which $\Delta f$ is the subcarrier space, $\operatorname{CP}\{\chi(n)\}$ represents the $\mathrm{CP}$ adding operation and $\operatorname{DAC}\{\chi(n)\} \triangleq \chi(t)$ where ideal DAC is assumed. Because of the assumption in Sec. I, it seems that the SI can be mitigated completely at the RF stage by using the AARFSIC if the emulating parameters can be obtained perfectly, i.e. $\sqrt{P_{s i}^{\prime}}=\sqrt{P_{s i}}, \sqrt{L_{s i}^{\prime}}=\sqrt{L_{s i}}$ and $\widetilde{H}_{s i}[k]=H_{s i}[k]$. However, our studies do not go into that direction as what we thought.

We can equivalently analyze and study AARFSIC in the baseband domain because of the assumption in Sec. I. After digitizing the $\mathrm{BB}$ analog residual self-interference, $r_{m, s i}^{R}(t)$, by using analog-to-digital converter (ADC), the baseband discrete-time-domain signal, $r_{m, s i}^{R}(n)$, can be obtained as

$$
\begin{aligned}
r_{m, s i}^{R}(n)= & \sqrt{P_{s i}} \sqrt{L_{s i}} \sqrt{P_{\mathrm{LNA}}} \operatorname{CP}\left\{\operatorname{IDFT}\left\{X_{m, s i}[k]\right\}\right\} \otimes h_{s i}(n) \\
& -\sqrt{P_{s i}^{\prime}} \sqrt{L_{s i}^{\prime}} \sqrt{P_{\mathrm{LNA}}} \operatorname{CP}\left\{\operatorname{IDFT}\left\{X_{m, s i}[k] \widetilde{H}_{s i}[k]\right\}\right\}
\end{aligned}
$$

where $P_{\mathrm{LNA}}$ represents the amplified power level of the receiver LNA, $\otimes$ denotes circular convolution and $h_{s i}(n)$ is the equivalent baseband expression of the wireless SI channel $h_{s i}(t)$.

Here comes the key question, if $\operatorname{CP}\left\{\operatorname{IDFT}\left\{X_{m, s i}[k]\right\}\right\} \otimes$ $h_{s i}(n)=\operatorname{CP}\left\{\operatorname{IDFT}\left\{X_{m, s i}[k] \widetilde{H}_{s i}[k]\right\}\right\}$ for $n \in\left[1, N+N_{\text {guard }}\right]$ holds even under the condition that we can obtain a perfect SI channel estimation? In order to answer this question, we study two scenarios: (i) AARFSIC for Full-Duplex OFDM wireless with one-path SI channel; (2) AARFSIC for FullDuplex OFDM wireless with multi-path SI channel.

\section{A. AARFSIC for One-Path Full-Duplex OFDM Wireless}

In two-antenna Full-Duplex wireless [10], the power of the line-of-sight path dominates the SI channel due to the proximity between the transmit and receive antenna of the Full-Duplex radio node. Hence, it is reasonable to assume that the SI channel is one-path, which could be expressed by $h_{s i}^{S}(n)=h_{s i, 0} \delta\left(n-n_{0}\right)$ where $h_{s i, 0}$ and $n_{0}$ represent the complex magnitude and delay of the wireless SI channel. The frequency domain SI channel, $H_{s i}^{S}[k]$, could be expressed as

$$
\begin{aligned}
H_{s i}^{S}[k] & =\operatorname{DFT}\left\{h_{s i}^{S}(n)\right\} \\
& =h_{s i, 0} e^{-j 2 \pi \Delta f k n_{0}}, k \in[1, N]
\end{aligned}
$$


Let $\widetilde{H}_{s i}^{S}[k]=\tilde{h}_{s i, 0} e^{-j 2 \pi \Delta f k \tilde{n}_{0}}$, where $\tilde{h}_{s i, 0}$ and $\tilde{n}_{0}$ represent the estimate of the $h_{s i, 0}$ and $n_{0}$ respectively, denote the estimate of the real SI channel $H_{s i}^{S}[k]$. Even for single-path SI channel, the delay could be from 0 to a large value, depending on system design. For example, when the SI channel is a short line-of-sight wireless channel, the delay $n_{0}$ should be 0 . However, when the short line-of-sight path is suppressed completely and only one reflecting path exists, the delay $n_{0}$ should be at least 1 , i.e. $n_{0} \geq 1$.

1) $n_{0}=0$ : When $\tilde{n}_{0}=n_{0}=0$ and after replacing $h_{s i}(n)$ and $\widetilde{H}_{s i}[k]$ in Eq. (2) by $h_{s i, 0}$ and $\tilde{h}_{s i, 0}$ respectively, the residual SI, $r_{m, s i}^{R}(n)$, could be expressed as

$$
\begin{aligned}
r_{m, s i}^{R}(n)= & \sqrt{P_{s i}} \sqrt{L_{s i}} \sqrt{P_{\mathrm{LNA}}} x_{m, s i}^{T}(n) h_{s i, 0} \\
& -\sqrt{P_{s i}^{\prime}} \sqrt{L_{s i}^{\prime}} \sqrt{P_{\mathrm{LNA}}} x_{m, s i}^{T}(n) \tilde{h}_{s i, 0} \\
\stackrel{a}{=} & 0
\end{aligned}
$$

2) $n_{0} \geq 1$ : When $n_{0} \geq 1$, the received baseband discretetime-domain SI signal can be expressed as

$$
\begin{aligned}
x_{m, s i}^{R}(n) & =\sqrt{P_{s i}} \sqrt{L_{s i}} \sqrt{P_{\mathrm{LNA}}} x_{m, s i}^{T}(n) \otimes h_{m, s i}^{S}(n) \\
& =\sqrt{P_{s i}} \sqrt{L_{s i}} \sqrt{P_{\mathrm{LNA}}} h_{s i, 0} x_{m, s i}^{T}\left(n-n_{0}\right)
\end{aligned}
$$

While the baseband discrete-time-domain cancellation signal can be derived and represented by

$$
x_{m, c}^{T}(n)=\sqrt{P_{s i}^{\prime}} \sqrt{L_{s i}^{\prime}} \sqrt{P_{\mathrm{LNA}}} \tilde{h}_{s i, 0} x_{m, s i}^{T}(n)
$$

Then, synchronizing the cancellation signal with the received SI signal via delaying $x_{m, c}^{T}(n)$ by $\tilde{n}_{0}$. Then, we can obtain the baseband cancellation signal at the receiver side as

$$
x_{m, c}^{R}(n)=\sqrt{P_{s i}^{\prime}} \sqrt{L_{s i}^{\prime}} \sqrt{P_{\text {LNA }}} \tilde{h}_{s i, 0} x_{m, s i}^{T}\left(n-\tilde{n}_{0}\right)
$$

The residual SI after the AARFSIC can be calculated as

$$
\begin{aligned}
r_{m, s i}^{R}(n) & =x_{m, s i}^{R}(n)-x_{m, c}^{R}(n) \\
& \stackrel{b}{=}
\end{aligned}
$$

$a$ and $b$ hold when perfect CSI of the SI channel can be obtained. That means the Eq. $\operatorname{CP}\left\{\operatorname{IDFT}\left\{X_{m, s i}[k]\right\}\right\} \otimes \tilde{h}_{s i}(n)=$ $\operatorname{CP}\left\{\operatorname{IDFT}\left\{X_{m, s i}[k] \widetilde{H}_{s i}[k]\right\}\right\}$ for $n \in\left[1, N+N_{\text {guard }}\right]$ holds true when perfect SI channel estimation can be obtained. Therefore, the SI could be mitigated completely by using AARFSIC. However, when the SI channel estimation is influenced by the receiver thermal noise, the strong SI could only be brought down to noise floor by AARFSIC [10] [11]. These study results are confined to anechoic Full-Duplex OFDM wireless.

\section{B. AARFSIC for Multi-Path Full-Duplex OFDM Wireless}

In more general Full-Duplex wireless, the SI channel, with high Ricean $K$ factor [5], consists of one direct path and multiple reflecting paths. Let $h_{s i}^{M}(n)=\sum_{p=0}^{P-1} h_{s i, p} \delta\left(n-n_{p}\right)$, where $h_{s i, p}$ and $n_{p}$ represent the complex magnitude and delay of the $p^{t h}$ path respectively, denote a $P$-path SI channel. The frequency domain SI channel, $H_{s i}^{M}[k]$, could be expressed as

$$
\begin{aligned}
H_{s i}^{M}[k] & =\operatorname{DFT}\left\{h_{s i}^{M}(n)\right\} \\
& =\sum_{p=0}^{P-1} h_{s i, p} e^{\jmath 2 \pi \Delta k n_{p}}, k \in[1, N]
\end{aligned}
$$

Without loss of generality, we choose $P=2$, which means the SI channel is composed of one direct path and one reflecting path, to simplify the analysis of the signal model. Hence, the SI channel can be rewrote as $H_{s i}^{M}[k]=h_{s i, 0} e^{-\jmath 2 \pi \Delta f k n_{0}}+h_{s i, 1} e^{-\jmath 2 \pi \Delta f k n_{1}}$. With the estimated CSI, $\widetilde{H}_{s i}^{M}[k]=H_{s i}^{M}[k]$, of the SI channel available, the RF cancellation signal, $x_{R F, m, c}^{R}(t)=$ $\sqrt{P_{s i}^{\prime}} \sqrt{L_{s i}^{\prime}} \operatorname{DAC}\left\{\operatorname{CP}\left\{\operatorname{IDFT}\left\{X_{m, s i}[k] \widetilde{H}_{s i}^{M}[k]\right\}\right\}\right\} e^{j 2 \pi f_{c} t}$, could be crafted. Then, the AARFSIC can be implemented as in Eq. (1). This is the current AARFSIC implementation procedure even for the multi-path Full-Duplex OFDM wireless. However, the residual SI after this cancellation is still very strong, which is shown in Fig. 2. Fortunately, we can equivalently obtain and analyze the over-the-air SI signal and the cancellation signal in the discrete time domain when the transceiver RF frontends are assumed ideal, which provides us the opportunity to insight the residual SI in discrete time domain.

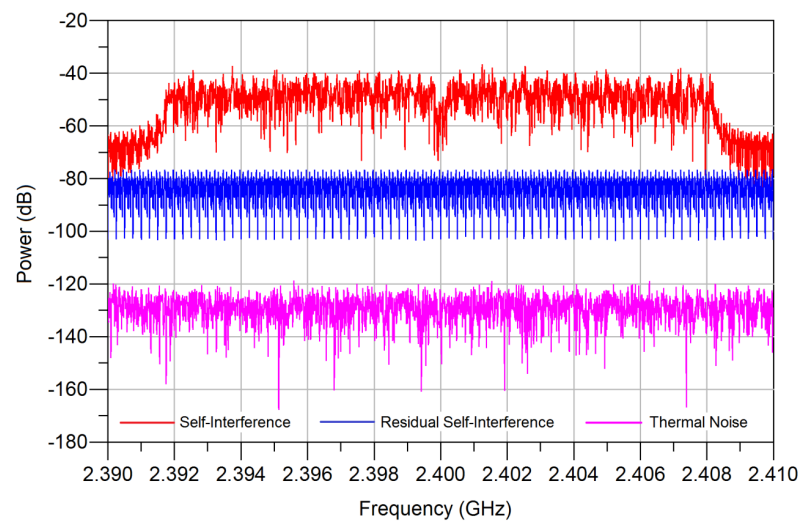

Fig. 2. When the Full-Duplex OFDM wireless with multipath (here 2 paths SI wireless channel is considered, the second path has 50ns time delay and $20 \mathrm{~dB}$ attenuation compared to the first path) SI channel, the AARFSIC has very limited capability of SIC. As it is shown, the power of the strong SI at the antenna of the receiver is $80 \mathrm{~dB}$ higher than the thermal noise. The AARFSIC can only achieve around $36 \mathrm{~dB}$ SIC. Therefore, there is still $44 \mathrm{~dB}$ residual SI.

1) Signal Model of the Received Self-Interference: After experiencing the multipath SI channel, the received SI can be expressed as $x_{m, s i}^{R}(n)=\sqrt{P_{s i}} \sqrt{L_{s i}} \sqrt{P_{\text {LNA }}} x_{m, s i}^{T}(n) \otimes h_{s i}^{M}(n)$. For simplifying the expression, we define $A_{s i} \triangleq \sqrt{P_{s i}} \sqrt{L_{s i}} \sqrt{P_{\mathrm{LNA}}}$. Then, the $m^{\text {th }}(m>1)$ OFDM symbol of the received SI, $x_{m, s i}^{R}(n)$, could be expressed in another form as

$$
x_{m, s i}^{R}(n)=\left\{\begin{array}{l}
A_{s i}\left[x_{m, s i}^{T}(n) h_{s i, 0}+x_{m-1, s i}^{T}\left(N+N_{\text {guard }}-n_{1}\right.\right. \\
\left.+n) h_{s i, 1}\right], \text { for } n \in\left[1, n_{1}\right] \\
A_{s i}\left[x_{m, s i}^{T}(n) h_{s i, 0}+x_{m, s i}^{T}\left(n-n_{1}\right) h_{s i, 1}\right], \\
\text { for } n \in\left[n_{1}+1, N+N_{\text {guard }}\right] .
\end{array}\right.
$$

2) Signal Model of the Crafted Cancellation Signal: The AARFSIC utilizes a $N$-size IDFT block to modulate the symbol block $X_{m, c}[k]=X_{m, s i}[k] \widetilde{H}_{m, s i}^{M}[k], k \in[1, N]$ where $\widetilde{H}_{s i}^{M}[k]=\tilde{h}_{s i, 0} e^{-\jmath 2 \pi \Delta f k \tilde{n}_{0}}+\tilde{h}_{s i, 1} e^{-\jmath 2 \pi \Delta f k \tilde{n}_{1}}$ is the estimate of $H_{m, s i}^{M}[k]$. The output of the IDFT block can be expressed 
as a linear combination of the two-path OFDM symbols as

$$
\begin{aligned}
x_{m, c}(n)= & \operatorname{IDFT}\left\{X_{m, c}[k]\right\} \\
= & \tilde{h}_{s i, 0} \operatorname{IDFT}\left\{X_{m, s i}[k] e^{-\jmath 2 \pi \Delta f k \tilde{n}_{0}}\right\} \\
& +\tilde{h}_{s i, 1} \operatorname{IDFT}\left\{X_{m, s i}[k] e^{-\jmath 2 \pi \Delta f k \tilde{n}_{1}}\right\} \\
= & x_{m, c}^{1}(n)+x_{m, c}^{2}(n), n \in[1, N]
\end{aligned}
$$

where $x_{m, c}^{1}(n)$ and $x_{m, c}^{2}(n)$ denote the first path and the second path of the cancellation OFDM symbol respectively, which are corresponding to cancel the first path and the second path of the received SI OFDM symbol. Because of $\tilde{n}_{0}=n_{0}=0$, the first path OFDM symbol could be represented by

$$
x_{m, c}^{1}(n)=x_{m, s i}(n) \tilde{h}_{s i, 0}, n \in[1, N]
$$

While with respect to $x_{m, c}^{2}(n)$, which can be regarded as the $m^{t h} N$-size OFDM symbol experiencing the second path of the SI channel with $\tilde{n}_{1}$ delay, it can be expressed as

$$
x_{m, c}^{2}(n)=\left\{\begin{array}{l}
x_{m, s i}\left(N-\tilde{n}_{1}+n\right) \tilde{h}_{s i, 1}, \text { for } n \in\left[1, \tilde{n}_{1}\right] \\
\left.x_{m, s i}\left(n-\tilde{n}_{1}\right) \tilde{h}_{s i, 1}\right], \text { for } n \in\left[\tilde{n}_{1}+1, N\right]
\end{array}\right.
$$

Therefore, the $m^{t h}$ cancellation OFDM symbol, $x_{m, c}(n)$, could be rewritten as

$$
x_{m, c}(n)=\left\{\begin{array}{l}
x_{m, s i}(n) \tilde{h}_{s i, 0}+x_{m, s i}\left(N-\tilde{n}_{1}+n\right) \tilde{h}_{s i, 1} \\
n \in\left[1, \tilde{n}_{1}\right] \\
\left.x_{m, s i}(n) \tilde{h}_{s i, 0}+x_{m, s i}\left(n-\tilde{n}_{1}\right) \tilde{h}_{s i, 1}\right] \\
n \in\left[\tilde{n}_{1}+1, N\right]
\end{array}\right.
$$

Then, the last $N_{\text {guard }}$ sample of OFDM symbol $x_{m, c}(n), n \in$ $[1, N]$ are copied and placed at the beginning of this OFDM symbol, which yields the CP added OFDM symbol, $x_{m, c}^{T}(n)$.

The BB cancellation signal can be represented by $x_{m, c}^{R}(n)=$ $\sqrt{P_{s i}^{\prime}} \sqrt{L_{s i}^{\prime}} \sqrt{P_{\mathrm{LNA}}} \mathrm{CP}\left\{\operatorname{IDFT}\left\{X_{m, s i}[k] \widetilde{H}_{s i}^{M}[k]\right\}\right\}$. Here, we define $A_{c}=\sqrt{P_{s i}^{\prime}} \sqrt{L_{s i}^{\prime}} \sqrt{P_{\mathrm{LNA}}}$. Then, the cancellation signal $x_{m, c}^{R}(n)$ can also be represented in another form as

$$
x_{m, c}^{R}(n)=\left\{\begin{array}{l}
A_{c}\left[x_{m, s i}^{T}(n) \tilde{h}_{s i, 0}+x_{m, s i}^{T}\left(N-\tilde{n}_{1}+n\right) \tilde{h}_{s i, 1}\right], \\
\text { for } n \in\left[1, \tilde{n}_{1}\right] ; \\
A_{c}\left[x_{m, s i}^{T}(n) \tilde{h}_{s i, 0}+x_{m, s i}^{T}\left(n-\tilde{n}_{1}\right) \tilde{h}_{s i, 1}\right], \\
\text { for } n \in\left[\tilde{n}_{1}+1, N+N_{\text {guard }}\right] .
\end{array}\right.
$$

Comparing the Eq. (10) and (15), we can find that the equation $\operatorname{CP}\left\{\operatorname{IDFT}\left\{X_{m, s i}[k]\right\} \otimes h_{s i}(n)\right\}=$ $\mathrm{CP}\left\{\operatorname{IDFT}\left\{X_{m, s i}[k] \widetilde{H}_{s i}[k]\right\}\right\}$ holds true for $n \in\left[n_{1}, N+N_{\text {guard }}\right]$, while it does not do for $n \in\left[1, n_{1}\right]$ even when transceiver RF front-ends are ideal and perfect CSI of the SI channel can be obtained. Now, we find the real problem preventing the AARFSIC to cancel the multi-path SI completely.

\section{Active Digital SIC IN Time Domain}

We discovered that the residual SI in discrete time domain, $r_{m, s i}^{R}(n)=x_{m, s i}^{R}(n)-x_{m, c}^{R}(n)$, is closely related to the transmitted symbols, $x_{m, s i}^{T}(n)$, which knowledge is available to the Full-Duplex radio node. Therefore, we propose to cancel the residual SI in the discrete time domain, which is as shown in Fig. 3. The factor, $A_{c}=\sqrt{P_{s i}^{\prime}} \sqrt{L_{s i}^{\prime}} \sqrt{P_{\mathrm{LNA}}}$ which is the estimate of the transmission factor $A_{s i}=\sqrt{P_{s i}} \sqrt{L_{s i}} \sqrt{P_{\mathrm{LNA}}}$, is required to implement the DSICT.

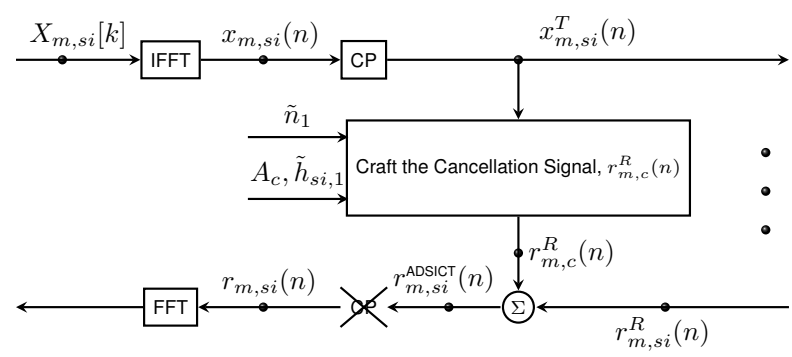

Fig. 3. The block diagram of DSICT.

With $A_{c}$ and the estimated $\tilde{h}_{s i, 1}, \tilde{n}_{1}=n_{1}$ available, the baseband cancellation signal, $r_{m, c}^{R}(n)$, could be crafted as

$$
r_{m, c}^{R}(n)=\left\{\begin{array}{l}
A_{c} \tilde{h}_{s i, 1}\left[x_{m-1, s i}^{T}\left(N+N_{\text {guard }}-n_{1}+n\right)\right. \\
\left.-x_{m, s i}^{T}\left(N-n_{1}+n\right)\right], \text { for } n \in\left[1, n_{1}\right] \\
0, \text { for } n \in\left[n_{1}+1, N+N_{\text {guard }}\right] .
\end{array}\right.
$$

Then, the DSICT can be implemented as

$$
\begin{aligned}
r_{m, s i}^{\mathrm{DSICT}}(n)= & r_{m, s i}^{R}(n)-r_{m, c}^{R}(n) \\
= & \left\{\begin{array}{l}
\left(A_{s i} h_{s i, 1}-A_{c} \tilde{h}_{s i, 1}\right)\left[x_{m-1, s i}^{T}(N+\right. \\
\left.N_{\text {guard }}-n_{1}+n\right)-x_{m, s i}^{T}\left(N-n_{1}\right. \\
+n)], \text { for } n \in\left[1, n_{1}\right] ; \\
0, \text { for } n \in\left[n_{1}+1, N+N_{\text {guard }}\right] .
\end{array}\right.
\end{aligned}
$$

where we can see that the residual SI can be eliminated completely by the proposed DSICT if the ideal estimate of $A_{s i}$ and $h_{s i, 1}$ can be obtained, i.e. $A_{c}=A_{s i}$ and $\tilde{h}_{s i, 1}=h_{s i, 1}$.

Perfect channel estimation is impossible in practical wireless communications, while it is possible to guarantee $A_{c}=A_{s i}$. Then, the residual SI after DSICT can be modeled as

$$
r_{m, s i}^{\mathrm{DSICT}}(n)=\left\{\begin{array}{l}
A_{s i} \Delta h_{s i, 1}\left[x_{m-1, s i}^{T}\left(N+N_{\text {guard }}-n_{1}+n\right)\right. \\
\left.-x_{m, s i}^{T}\left(N-n_{1}+n\right)\right], \text { for } n \in\left[1, n_{1}\right] \\
0, \text { for } n \in\left[n_{1}+1, N+N_{\text {guard }}\right] .
\end{array}\right.
$$

where $\Delta h_{s i, 1}=h_{s i, 1}-\tilde{h}_{s i, 1}$ denotes the estimate error of $h_{s i, 1}$. The amount of SIC DSICT achieve can be computed as

$$
\begin{aligned}
\alpha_{\mathrm{DSICT}}(d B) & =\mathrm{E}\left\{\left|r_{m, s i}(n)\right|^{2}\right\}_{d B}-\mathrm{E}\left\{\left|r_{m, s i}^{\mathrm{DSICT}}(n)\right|^{2}\right\}_{d B} \\
& =\mathrm{dB}\left[\frac{\mathrm{E}\left\{\left|h_{s i, 1}\right|^{2}\right\}}{\mathrm{E}\left\{\left|\Delta h_{s i, 1}\right|^{2}\right\}}\right]
\end{aligned}
$$

For the Full-Duplex OFDM wireless with two-path SI channel, the power of the residual SI after AARFSIC and DSICT is closely related to the channel estimation of the second path.

\section{NumERICAL RESUlTS AND Discussion}

In order to further confirm our findings and the proposed DSICT, system level design and simulation based on the softwares of ADS and Matlab is implemented. The transceiver RF front-end and baseband digital system are constructed on ADS and algorithm implementation is carried out on Matlab.

In the system level simulation, the power ratio of the SI to the thermal noise (INR) is $80 \mathrm{~dB}$. The system parameters are chosen from the $36 \mathrm{Mbps}$ radio link of the IEEE $802.11 \mathrm{~g}$. 


\section{A. Numerical Results}

Two-path wireless SI channel is chosen in this first study. The second path has $50 \mathrm{~ns}$ time delay $\left(n_{1}=1\right)$ and $20 \mathrm{~dB}$ attenuation compared to the first path. As it has been shown in Fig. 2, the AARFSIC could achieve about 36dB SIC for the Full-Duplex OFDM wireless. In order to calibrate the residual SI after the DSICT, a desired radio link is added and the bit error rate (BER) is used to measure the loss of the desired SNR due to the residual SI.

1) Ideal Transceiver RF Front-End and Perfect Channel Estimation: Even with ideal transceiver RF front-end and perfect channel estimation, the AARFSIC can not bring the strong SI down to the thermal noise floor. However, the proposed DSICT can mitigate the residual SI completely, which could be demonstrated by Fig. 4. The BER curve of the Full-Duplex IEEE $802.11 \mathrm{~g}$ overlap with that of the standard IEEE $802.11 \mathrm{~g}$, which means the total $80 \mathrm{~dB}$ SI is canceled completely by the combination of AARFSIC and DSICT.

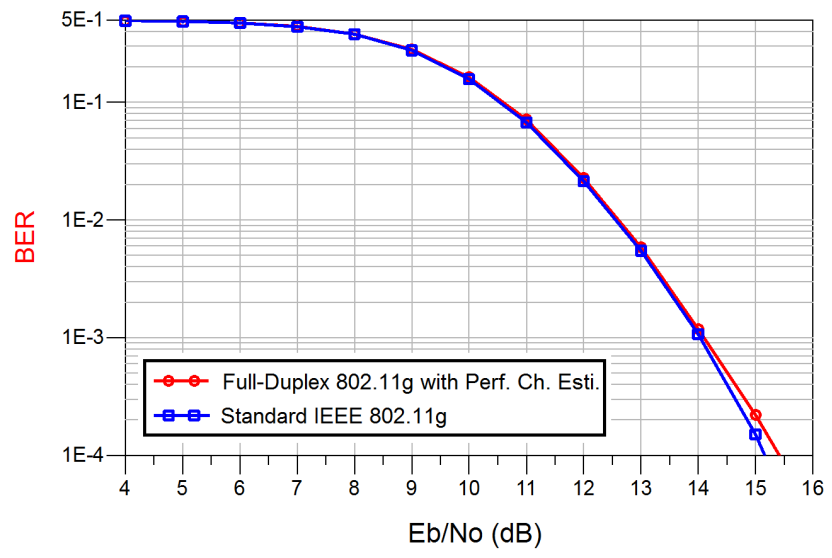

Fig. 4. Comparison of the BER curves between the Full-Duplex IEEE $802.11 \mathrm{~g}$ with perfect channel estimation and the standard IEEE $802.11 \mathrm{~g}$ system.

2) Ideal Transceiver RF Front-End and Real Channel Estimation: Here, the transceiver RF front-ends are still assumed ideal, while the real waveform channel estimation is considered. During the SI channel estimation, the receiver thermal noise is considered as the only interference. As it is shown in the Fig. 5, the channel estimation error due to the receiver thermal noise could cause around $3 \mathrm{~dB}$ residual SI. In another words, there is still around $3 \mathrm{~dB}$ residual SI after using the combination scheme of the AARFSIC and DSICT if the CSI is obtained by practical channel estimation in a standard IEEE 802.11g system.

\section{B. Discussion}

Although the two-path SI channel has been chosen for theoretical analysis and system level simulation in this paper, FullDuplex OFDM wireless with multi-path SI channel $(P>2)$ can also be studied using the AARFSIC and DSICT signal processing model presented in the paper. When CSI could be obtained perfectly, the strong SI can be canceled completely

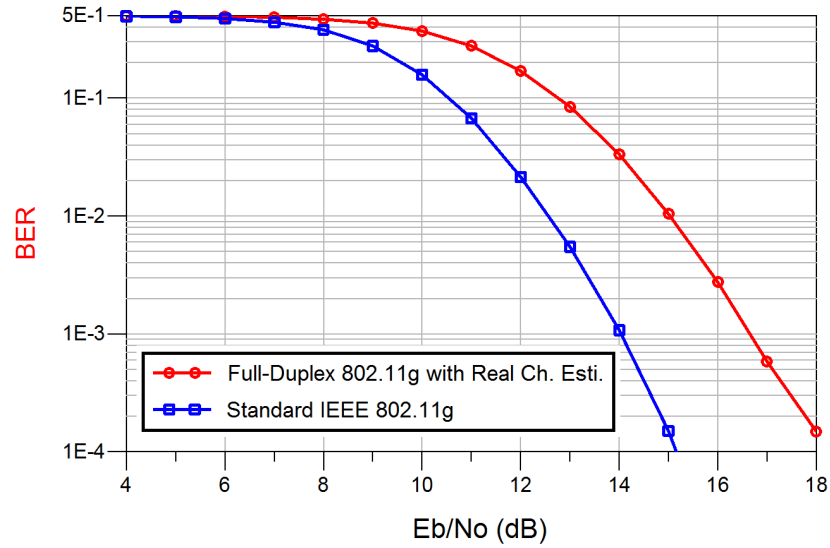

Fig. 5. Comparison of the BER curves between the Full-Duplex IEEE 802.11g with real channel estimation and the standard IEEE 802.11g system.

by using the combination scheme of AARFSIC and DSICT. But when in real channel estimation condition, there will be around $3 \mathrm{~dB}$ residual SI due to the receiver thermal noise.

\section{CONCLUSION}

The AARFSIC has been studied a lot. However, the core problem causing the untolerable residual self-interference behind the Full-Duplex OFDM wireless has not been completely fixed. We present the anatomy of the AARFSIC for the multipath Full-Duplex OFDM wireless and find the relationship between the residual self-interference and its own transmission in the discrete time domain. Then, we propose DSICT to complement AARFSIC for Full-Duplex OFDM wireless.

\section{REFERENCES}

[1] J. I. Choi, M. Jain, k. Srinivasan, P. Levis, and S. Katti, "Achieving single channel, Full Duplex wireless communications," in Proc. ACM MOBICOM, 2010.

[2] M. Jain, J. Choi, T. Kim, D. Bharadia, K. Srinivasan, P. Levis, S. Katti, P. Sinha, and S. Seth, "Practical real-time Full Duplex wireless," in Proc. ACM MOBICOM, 2011.

[3] M. Duarte and A. Sabharwal, "Full Duplex wireless communications using off-the-shelf radios: Feasibility and first results," in Proc. Asilomar Conference on Signals, Systems and Computers, 2010.

[4] M. Duarte, C. Dick, and A. Sabharwal, "Experiment driven characterization of Full-Duplex wireless communications," IEEE Transactions on Wireless Communications, vol. 11, no. 12, pp. 505-514, 2012.

[5] E. Everett, A. Sahai, and A. Sabharwal, "Passive self-interference suppression for Full-Duplex infrastructure nodes," IEEE Transactions on Wireless Communications, vol. 13, no. 2, pp. 680-694, 2014.

[6] D. Bharadia, E. McMilin, and S. Katti, "Full duplex radios," in Proc. ACM SIGCOM, 2013.

[7] H. Tech., "5G: A technology vision," Huawei Tech. Report, 2014.

[8] A. Sabharwal, P. Schniter, D. Guo, D.-W. Bliss, S. Rangarajan, and R. Wichman, "In-band Full-Duplex wireless: challenges and opportunities," http://arxiv.org/pdf/1311.0456.pdf.

[9] B. Kaufman, J. Lilleberg, and B. Aazhang, "Analog baseband cancellation for Full-Duplex: An experimental driven analysis," http://arxiv.org/pdf/1312.0522.pdf.

[10] Z. Zhan, G. Villemaud, and J.-M. Gorce, "Design and evaluation of a wideband Full-Duplex OFDM system based on AASIC," in Proc. IEEE 24th Annual International Symposium on Personal, Indoor and Mobile Radio Communications, 2013.

[11] _ "Analysis and reduction of the impact of thermal noise on the FullDuplex OFDM radio," in Proc. IEEE Radio and Wireless Symposium, 2014 